\title{
Factors associated with psychological distress of medical doctor in Kagawa prefecture, Japan: a pilot study
}

\author{
Takeshi Suzue $^{1}$, Noriko Sakano ${ }^{2}$, Yoshikazu Miyamae ${ }^{3}$, Takeshi Yoda ${ }^{1}$, Akira Yoshioka $^{1}$, Taichi \\ Nagatomi ${ }^{4}$, Wataru Shiraki ${ }^{5}$, Tomohiro Hirao ${ }^{1}$ \\ ${ }^{1}$ Department of Public Health, Faculty of Medicine, Kagawa University, Kagawa, Japan; \\ *Corresponding Author: suzue@med.kagawa-u.ac.jp \\ ${ }^{2}$ Department of Hygiene, Faculty of Medicine, Kagawa University, Kagawa, Japan; \\ ${ }^{3}$ Center for Educational Research and Teacher Development, Faculty of Education, Kagawa University, Kagawa, Japan; \\ ${ }^{4}$ Institute of Research Partnership Center for Social Collaboration and Intellectual Property, Kagawa University, Kagawa, Japan; \\ ${ }^{5}$ Department of Reliability-Based Information Systems Engineering, Faculty of Engineering, Kagawa University, Kagawa, Japan.
}

Received 6 September 2011; revised 15 November 2011; accepted 30 November 2011.

\begin{abstract}
Objective: We evaluated the psychological distress of medical doctor using a 6-item instrument (the K6) in Kagawa prefecture, Japan. Methods: A total of 284 medical doctors (236 men and 48 women) were analyzed in a cross-sectional investigation study. The association between psychological distress and clinical factors were evaluated by the $\mathrm{K} 6$ instrument, with psychological distress defined as $\mathbf{1 3}$ or more points out of a total of 24 points. Results: A total of 17 doctors $(6.0 \%)$ as defined as psychological distress. The significant relationships between the $\mathrm{K} 6$ score and age, experience duration as clinician were not noted. The $\mathrm{K} 6$ score in subjects with consciousness of suicide was significantly higher than that without. In addition, the K6 score in subjects without cooperation with specialists was higher than those without, but not at a significant level. Conclusions: Some factors i.e. consciousness of suicide and cooperation with specialists might be associated with psychological distress, as assessed by the $\mathrm{K} 6$ instrument, in medical doctor in Kagawa prefecture, Japan.
\end{abstract}

Keywords: Psychological Distress; The K6; Medical Doctor; Kagawa; Japan

\section{INTRODUCTION}

Mental health has become public health challenge in Japan. For example, over 30 thousand subjects suicide in a year for over 10 years [1]. About $14 \%$ of the global disease burden has been attributed to mental illness, mostly due to the chronically disabling nature of depression and other common mental disorders [2].

Recently, the demand for medical doctors has been increasing in various areas because of a medical reform bill and government amendments to the medical service payment structure in Japan. In some literatures, the psychological distress of medical doctors in Japan has reported [3,4]. However, the evaluation of psychological distress of medical doctors in Japan still remains to be investigated.

Therefore, we evaluated the psychological distress of medical doctors by using the K6 instrument, the scale of psychological distress, in Kagawa prefecture, Japan and its relation to clinical factors in this cross-sectional investigation study.

\section{SUBJECTS AND METHODS}

\subsection{Subjects}

We used data of 334 respondents (37.8\%) among 884 medical doctors (physician, surgeon and psychiatrist) in Kagawa prefecture, Japan, who met the following criteria; 1) they had completely answered the questionnaires (clinical characteristics and the K6); and 2) they provided informed consent. Therefore, we analyzed 284 (32.1\%) medical doctors (236 men and 48 women), aged $55.8 \pm 11.9$ years in this cross sectional investigation study.

Ethical approval for the study was obtained from the Ethical Committee of Faculty of Medicine, Kagawa University, Japan.

\subsection{Psychological Distress}

The K6 was used as an indicator of psychological dis- 
tress [8,9]. The 6 questions were as follows: "Over the last month, how often did you feel: 1) nervous, 2) hopeless, 3) restless or fidgety, 4) so sad that nothing could cheer you up, 5) that every thing was an effort, 6) worthless?”. Participants were asked to respond by choosing "all of the time" (4 points), "most of the time" (3 points), "some of the time" (2 points), "a little of the time" (1 point), and "none of the time" (0 points). Therefore, total point score ranged from 0 to 24 . The K6 has been developed using modern psychometric theory and has been shown to be superior to some existing scales in brevity and psychometric properties [5-7]. The Japanese version of the K6 has been recently developed, using the standard back-translation method, and has been validated [8]. As suggested by Kessler et al. [7], we classified participants with scores of 13 points or more as having psychological distress.

\subsection{Measurement of Clinical Variables}

The questionnaires for medical doctors consisted of the following details in sequence: age, sex, experience duration as clinician. In addition, we evaluated the consciousness of suicide in clinical practice, anxiety of preventing suicide and cooperation with specialist in their daily work.

\subsection{Statistical Analysis}

Data are expressed as mean \pm standard deviation (SD) values. A statistical analysis was performed using NOVA and Scheffe's F test. Simple correlation analysis was performed as well to test for the significance of the linear relationship among continuous variables, where $p<0.05$ was considered to be statistically significant.

\section{RESULTS}

The total K6 score was $4.3 \pm 3.9$, and details were as follows: 1 ) nervous: $1.1 \pm 1.0 ; 2$ ) hopeless: $0.4 \pm 0.7$; 3) restless or fidgety: $0.8 \pm 0.9 ; 4$ ) so sad that nothing could cheer you up: $0.7 \pm 0.8$; 5 ) that every thing was an effort: $0.8 \pm 0.9$; 6 ) worthless: $0.5 \pm 0.8$. A total of 17 medical doctors $(6.0 \%)$ as defined as psychological distress. We evaluated the relationship between the K6 score and age (Figure 1), experience duration as clinician. The significant relationships between the K6 score and age ( $\mathrm{r}=$ $-0.018, p=0.7663)$, and between the K6 score and experience duration as clinician $(\mathrm{r}=-0.011, p=0.8519)$ were not noted in this study.

In addition we evaluated the factors i.e. consciousness of suicide, anxiety of preventing suicide and cooperation with specialist (Table 1). The K6 score in subjects with consciousness of suicide (most of the time) was signifi- cantly higher than that without (a little of the time). In addition, the K6 score in subjects with cooperation with specialist (most of the time) was lower than that in sub- jects without that, but not at a significant level.

\section{DISCUSSION}

We firstly evaluated the psychological distress of medical doctors using the K6 instrument in Kagawa prefecture, Japan.

According to the link between psychological distress and medical doctors, Hayasaka et al. has reported that work environment factors, particularly night duty, play important roles in modulating psychological distress among female doctors by using the thirty-item version of the General Health Questionnaire (GHQ-30) [3,9]. Wada et al. also showed that depressive symptoms were indi-

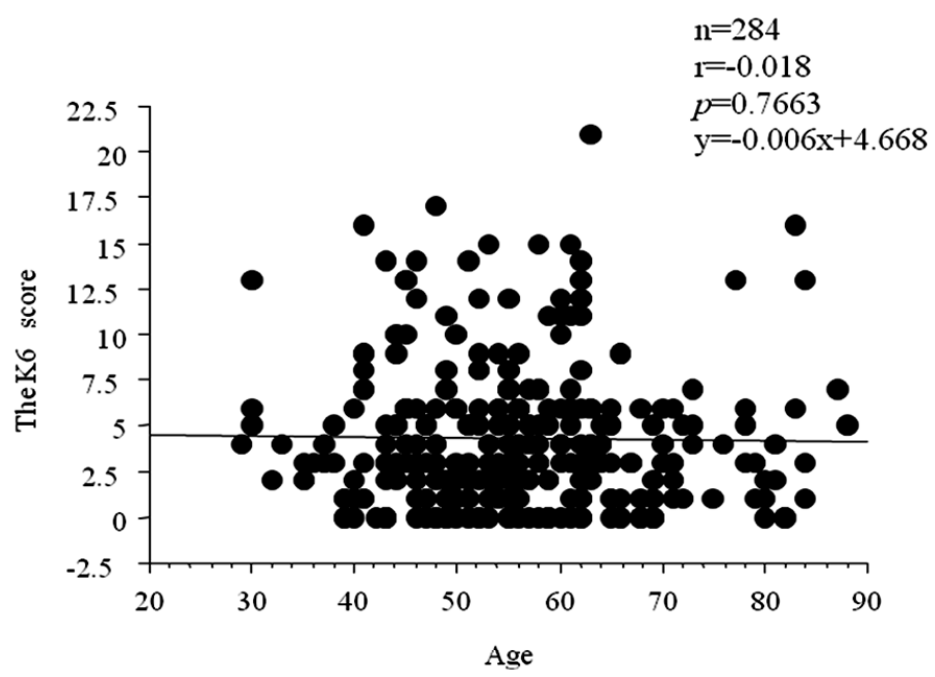

Figure 1. Relationship between the K6 score and age in medical doctor in Kagawa prefecture, Japan. 
Table 1. Relation between the K6 soore and clinical factors in medical doctor.

\begin{tabular}{|c|c|c|c|c|c|}
\hline & Most of the time & Some of the time & A little of the time & & None of the time \\
\hline \multicolumn{6}{|c|}{ Consciousness of suiside } \\
\hline Number of subjects & 7 & 124 & 132 & & 21 \\
\hline The K6 socre & $8.6 \pm 7.8$ & $4.7 \pm 3.8$ & $3.7 \pm 3.3$ & a & $5.0 \pm 5.4$ \\
\hline \multicolumn{6}{|c|}{ Anxiety of preventing suicide } \\
\hline Number of subjects & 111 & 153 & 16 & & 4 \\
\hline The K6 socre & $4.4 \pm 4.0$ & $4.3 \pm 3.9$ & $4.6 \pm 4.6$ & & $5.3 \pm 5.6$ \\
\hline \multicolumn{6}{|c|}{ Cooperation with specialist } \\
\hline Number of subjects & 21 & 115 & 101 & & 47 \\
\hline The K6 socre & $3.7 \pm 3.8$ & $4.6 \pm 3.9$ & $4.2 \pm 3.9$ & & $4.3 \pm 4.2$ \\
\hline
\end{tabular}

a: $p<0.05$ vs Most of the time.

cated in $8.3 \%$ of men and $10.5 \%$ of women, and $5.7 \%$ of men and 5.8\% women were determined to exhibit suicidal ideation using the Japanese version of the Quick Inventory of Depressive Symptomatology (QIDS) [4]. The number of unreasonable demands and complaints in the previous 6 months was significantly associated with depressive symptoms and suicidal ideation [4]. In this study, we evaluated the psychological distress of medical doctors evaluated by the K6 instrument in Kagawa prefecture, Japan. Consciousness of suicide and cooperation with specialist in their daily work might be associated with psychological distress in medical doctors in Kagawa prefecture, Japan. Therefore, it seems reasonable to suggest that simply supporting in medical doctors in mental health and suicide is also required in Kagawa prefecture, Japan.

In some literatures, psychological distress by using the K6 has been reported. Kuriyama et al. reported that they evaluated psychological distress of 43,716 communitydwelling people aged 40 years or older living in Japan [10]. Subjective social status seems to be a stronger predictor of psychological distress among both men and women in the Japanese community than traditional socioeconomic status [11]. Inoue et al. showed that part time workers in men and temporary/contract workers in women were associated with poor mental health in a total of 9461 men and 7777 women employees. No clear relation in mental health was noted in company size or occupation [12]. However, there is no report of psychological distress of medical doctors evaluated by the K6 instrument in Japan. In this study, by using the K6, we evaluated the psychological distress of medical doctors in Kagawa prefecture, Japan. A total of 17 medical doctors $(6.0 \%)$ was defined as psychological distress. The rate of psychological distress was similar to that previous report [10]. However, some factors in their daily work i.e. consciousness of suicide and cooperation with specialists might be associated with their psychological distress. This reference and pilot data obtained from this study might be useful for preventing psychological distress in medical doctors in Japan.

Potential limitations still remain in this study. First, our study was a cross sectional and not a longitudinal study. Second, 284 medical doctors among 884 doctors in our study voluntarily underwent measurement in Kagawa prefecture, Japan: they were therefore more likely to be health-conscious compared with the average medical doctors. The third, most of them were physicians and there was no psychiatrist. To show this, further prospective studies and in other prefectures are needed in the Japanese.

\section{ACKNOWLEDGEMENTS}

This research was supported in part by Kagawa prefecture, Japan.

\section{REFERENCES}

[1] Cabinet Office Government, Japan (2011). http://www8.cao.go.jp/jisatsutaisaku/whitepaper/w-2010/ pdf/gaiyou/pdf/g04.pdf,

[2] Prince, M., Patel, V., Saxena, S., Maj, M., Maselko, J., Phillips, M.R. and Rahman, A. (2007) No health without mental health. Lancet, 370, 859-877. doi:10.1016/S0140-6736(07)61238-0

[3] Hayasaka, Y., Nakamura, K., Yamamoto, M. and Sasaki, S. (2007) Work environment and mental health status assessed by the general health questionnaire in female Japanese doctors. Industrial Health, 45, 781-786. doi:10.2486/indhealth.45.781

[4] Wada, K., Yoshikawa, T., Goto, T., Hirai, A., Matsushima, E., Nakasahima, Y., Akaho, R., Kido, M. and Hosaka, T. (2010) Association of depression and suicidal ideation with unreasonable patient demands and complaints among Japanese physicians: A national cross-sectional survey. International Journal of Behavioral Medicine, 18, 384-390. doi:10.1007/s12529-010-9132-7

[5] Kessler, R.C., Andrews, G., Colpe, L.J., Hiripi, E., Mroczek, D.K., Normand, S.L., Walters, E.E. and Zaslavsky, A.M. (2002) Short screening scales to monitor population prevalences and trends in non-specific psychological distress. Psychological Medicine, 32, 959-976. 
doi:10.1017/S0033291702006074

[6] Furukawa, T.A., Kessler, R.C., Slade, T. and Andrews, G. (2003) The performance of the K6 and K10 screening scales for psychological distress in the Australian National Survey of Mental Health and Well-Bing. Psychological Medicine, 33, 357-362. doi:10.1017/S0033291702006700

[7] Kessler, R.C., Barker, P.R., Colpe, L.J., Epstein, J.F., Gfroerer, J.C., Hiripi, E., Howes, M.J., Normand, S.L., Manderscheid, R.W., Walters, E.E. and Zaslavsky, A.M. (2003) Screening for serious mental illness in the general population. Archives of General Psychiatry, 60, 184-189. doi:10.1001/archpsyc.60.2.184

[8] Furukawa, T.A., Kawakami, N., Saitoh, M., Ono, Y., Nakane, Y., Nakamura, Y., Tachimori, H., Iwata, N., Uda, H., Nakane, H., Watanabe, M., Naganuma, Y., Hata, Y., Kobayashi, M., Miyake, Y., Takeshima, T. and Kikkawa, $\mathrm{T}$. (2008) The performance of the Japanese version of the K6 and K10 in the World Mental Health Survey Japan. International Journal of Methods in Psychiatric Research, 17, 152-158.

doi:10.1002/mpr.257

[9] Iwata, N., Uno, B. and Suzuki, T. (1994) Psychometric properties of the 30-item version general health questionnaire in Japanese. Japanese Journal of Psychiatry \& Neurology, 48, 547-556.

[10] Kuriyama, S., Nakaya, N., Ohmori-Matsuda, K., Shimazu, T., Kikuchi, N., Kakizaki, M., Sone, T., Sato, F. Nagai, M., Sugawara, Y., Akhter, M., Higashiguchi, M., Fukuchi, N., Takahashi, H., Hozawa, A. and Tsuji, I. (2009) Factors associated with psychological distress in a community dwelling Japanese population: the Ohsaki Cohort 2006 Study. Journal of Epidemiology, 19, 294302. doi:10.2188/jea.JE20080076

[11] Sakurai, K., Kawakami, N., Yamaoka, K., Ishikawa, H. and Hashimoto, H. (2010) The impact of subjective and objective social status on psychological distress among men and women in Japan. Social Science \& Medicine, 70, 1832-1839. doi:10.1016/j.socscimed.2010.01.019

[12] Inoue, A., Kawakami, N., Tsuchiya, M., Sakurai, K. and Hashimoto, H. (2010) Association of occupation, employment contract, and company size with mental health in a national representative sample of employees in Japan. Journal of Occupational Health, 52, 227-240. doi:10.1539/joh.O10002 